Running head: VARIABILITY IN TRAJECTORIES IN CROSS-LAGGED MODELS

\title{
Accounting for Time-Varying Inter-Individual Differences of Trajectories when assessing
} Cross-lagged Models

\author{
Paul Wesley Scott \\ University of Pittsburgh
}

This manuscript is an unabridged, rough edit earlier version.

The Version of Record (VoR) for this manuscript has been published and is available in Structural Equation Modeling: A Multi-Disciplinary Journal, (29 September 2020) https://www.tandfonline.com/doi/full/10.1080/10705511.2020.1819815 


\title{
Title: Accounting for Time-Varying Inter-Individual Differences in Trajectories when assessing Cross-Lagged Models
}

\begin{abstract}
This paper explores relationships amongst cross-lagged models allowing trajectories to be freely estimated, some accounting for time-varying differences amongst individuals (Autoregressive Latent Trajectory (ALT), General Cross-lagged Model (GCLM), and Latent Growth Curve Model with Structured Residuals and Unspecified Growth Trajectory (LGCM-SR-UGT)) and some not (Cross-lagged Panel Model (CLPM), Random Intercept Cross-lagged Panel Model (RICLPM), and Mean Stationary GCLM). An applied example using LSAY data demonstrates these models. Simulations examine (1) fit indices assessing "good" fit and Bayes Factor for model selection; (2) consequences of ignoring variability in trajectories on cross-lagged estimates. Findings were (1) RMSEA discerned "good" fit and Bayes Factor tended to select models closely related to true model over less related models; (2) various patterns of bias in path estimates and standard errors are found, in particular, causal dominance in conjunction with time-variant between-person variance and covariance were notably influential on bias in path estimates.
\end{abstract}

Keywords: cross-lagged panel model, general cross-lagged model, random intercept crosslagged panel model, autoregressive latent trajectory model, latent growth curve model with structured residuals 


\section{Introduction}

The Cross-Lagged Panel Model (CLPM) has become a standard for exploring bidirectional relationships amongst different constructs over time (Jöreskog, 1970; Jöreskog \& Sörbom, 1979). Especially for assessing causal relationships between constructs (i.e., Granger Causality, Granger, 1980; Granger, 1969). If we establish a CLPM where standing at a given time has a direct dependence on the prior time point alone (i.e., lag 1) both within a process and between processes, then we have a CLPM with first-order autoregressive (i.e., AR(1)) and first-order cross-lagged (i.e., CL(1)) structure. Ignoring measurement error, these models may be set up by either centering individuals around a time-varying group level mean, then fitting the lagged relations to the individuals' time-specific deviations (aka residuals, innovations, impulses, shocks, or perturbations) as represented in models 1a-d; or by fitting lagged relations directly to observed variables as depicted in models $2 \mathrm{a}-\mathrm{b}$ :

$$
\begin{gathered}
y_{i t}=\mu_{t}^{(y)}+\epsilon_{i t}^{(y)} \\
x_{i t}=\mu_{t}^{(x)}+\epsilon_{i t}^{(x)} \\
\epsilon_{i t}^{(y)}=\rho_{y y} \epsilon_{i t-1}^{(y)}+\rho_{y x} \epsilon_{i t-1}^{(x)}+u_{i t}^{(y)} \\
\epsilon_{i t}^{(x)}=\rho_{x x} \epsilon_{i t-1}^{(x)}+\rho_{x y} \epsilon_{i t-1}^{(y)}+u_{i t}^{(x)}
\end{gathered}
$$

The formulation above (1a-d) allows the group-level overall changes across time to be captured through the mean structures, $\mu_{t}^{(y)} \& \mu_{t}^{(x)}$. The moment to moment carry-over effect of individuals' time-specific deviations from the group-level trajectory within process are captured by the autoregressive parameters $\rho_{x x} \& \rho_{y y}$, and between process through the cross-lagged 
parameters $\rho_{x y} \& \rho_{y x}$. The time specific error-variance for each process co-vary with one another, $\operatorname{Cov}\left(u_{i t}^{(x)}, u_{i t}^{(y)}\right)$, allowing instantaneous relations amongst the processes to be captured.

$$
\begin{aligned}
& y_{i t}=\alpha_{t}^{(y)}+\rho_{y y} y_{i t-1}+\rho_{y x} x_{i t-1}+u_{i t}^{(y)} \\
& x_{i t}=\alpha_{t}^{(x)}+\rho_{x x} x_{i t-1}+\rho_{x y} y_{i t-1}+u_{i t}^{(x)}
\end{aligned}
$$

The above models (2a-b) allow time-specific influences on a process to have a common impact on individuals by fitting time-specific intercept terms, $\alpha_{t}^{(x)} \& \alpha_{t}^{(y)}$. The time-specific deviations of individuals are captured by the impulses, $u_{i t}^{(y)} \& u_{i t}^{(x)}$, though the impulses are independent of one another, the influence of these impulses over time within a process are captured in the autoregressive paths, $\rho_{y y} \& \rho_{x x}$, and between processes through the cross-lagged paths, $\rho_{y x} \& \rho_{x y}$, with instantaneous time-specific relationships between processes being captured by the covariance of implulses, $\operatorname{Cov}\left(u_{i t}^{(x)}, u_{i t}^{(y)}\right)$. In fitting the autoregressive and crosslagged paths to the observed variables, the overall group-level trajectory is captured through a combination of time-specific common effects with the autoregressive and cross-lagged paths. Namely, the mean structure across time points would be estimated in an accumulative fashion as such:

$$
\mu_{t}^{(y)}=\alpha_{t}^{(y)}+\rho_{y y} \mu_{t-1}^{(y)}+\rho_{y x} \mu_{t-1}^{(x)} \quad \mu_{t}^{(x)}=\alpha_{t}^{(x)}+\rho_{x x} \mu_{t-1}^{(x)}+\rho_{x y} \mu_{t-1}^{(y)}
$$

If we assume autoregressive and cross-lagged paths are constant over time, we can express equation 3 compactly as: 


$$
\begin{aligned}
& \mu_{t}^{(y)}=\sum_{h=t-1}^{h=0}\left(\rho_{y y}^{h} \alpha_{t-h}^{(y)}\right)+\rho_{y x} \alpha_{t-1}^{(x)}+\sum_{h=t-1}^{h=2}\left(\left[\rho_{y y}^{(h)} \rho_{y x}^{(h+1)}\right] \alpha_{t-h}^{(x)}\right)+ \\
& \sum_{h=t-1}^{h=2}\left(\left[\rho_{x x}^{(h+1)} \rho_{y x}^{(h)}\right] \alpha_{t-h}^{(x)}\right)+\sum_{h=t-1}^{h=2}\left(\left[\rho_{x y}^{(h+1)} \rho_{y x}^{(h)}\right] \alpha_{t-h}^{(y)}\right) \\
& \mu_{t}^{(x)}=\sum_{h=t-1}^{h=0}\left(\rho_{x x}^{h} \alpha_{t-h}^{(x)}\right)+\rho_{x y} \alpha_{t-1}^{(y)}+\sum_{h=t-1}^{h=2}\left(\left[\rho_{x x}^{(h)} \rho_{x y}^{(h+1)}\right] \alpha_{t-h}^{(y)}\right)+ \\
& \sum_{h=t-1}^{h=2}\left(\left[\rho_{y y}^{(h+1)} \rho_{x y}^{(h)}\right] \alpha_{t-h}^{(y)}\right)+\sum_{h=t-1}^{h=2}\left(\left[\rho_{y x}^{(h+1)} \rho_{x y}^{(h)}\right] \alpha_{t-h}^{(x)}\right)
\end{aligned}
$$

Where $h$ pertains to lags, with $h=0$ being contemporary with time point $t$ (i.e., no lags behind), $h=1$ referring to the prior time point $t$ - 1 (i.e., one lag behind), and so on; $t$ - $h$ captures a time point relative to the lags it is behind another time point, so a lag of order $t-1$ is the maximal lags behind a given time point. For example, at time point $t=6, h=6-1=5$ lags behind, setting us at time point $t-h=6-5=1$. For path product terms above, e.g., $\left[\rho_{y x}^{(h+1)} \rho_{x y}^{(h)}\right]$, the parenthetic $(h)$ and $(h+1)$ superscripted terms refer to AR \& CL path coefficients from lag $h$ and the lag immediately prior to lag $h$, lag $h+1$; while the non-parenthesized superscript $h$ associated with the AR paths refer to an exponent value corresponding to a certain lag value.

Occasion effects within a given process have contemporaneous, direct, and indirect influences on the mean structure of that process. Hence the influence of a process on itself will be present from lag orders $\mathrm{h}=\mathrm{t}-1$ to $\mathrm{h}=0$ and carried through the autoregressive paths, with the 
contemporaneous influence being $\rho_{x x}^{0}=1$ or $\rho_{y y}^{0}=1$ the direct influence from the prior time point being $\rho_{x x}^{1}=\rho_{x x}$ or $\rho_{y y}^{1}=\rho_{y y}$ and the indirect effects for each further lag behind (from $h=t-1$ to $h=2$ ) either exponentially decreasing (when $-1<\mathrm{AR}<1$ ), increasing (when $|\mathrm{AR}|>1$ ), or persisting without decay (when $|\mathrm{AR}|=1)$. This is expressed as $\sum_{h=t-1}^{h=0}\left(\rho_{y y}^{h} \alpha_{t-h}^{(y)}\right)$ for y \& $\sum_{h=t-1}^{h=0}\left(\rho_{x x}^{h} \alpha_{t-h}^{(x)}\right)$ for $\mathrm{x}$. Indirect effects of a process on itself also come in the form of a composite of cross-lagged paths (i.e., $\sum_{h=t-1}^{h=2}\left(\left[\rho_{x y}^{(h+1)} \rho_{y x}^{(h)}\right] \alpha_{t-h}^{(y)}\right)$ for y $\&$ $\sum_{h=t-1}^{h=2}\left(\left[\rho_{y x}^{(h+1)} \rho_{x y}^{(h)}\right] \alpha_{t-h}^{(x)}\right)$ for $\left.\mathrm{x}\right)$, which could be understood as a process' mediated influence on itself via the other process.

Occasions effects of one process have a single direct influence from lag $h=1$ (i.e., $\rho_{x y} \alpha_{t-1}^{(y)}$ for $\mathrm{y}$ on $\mathrm{x} \& \rho_{y x} \alpha_{t-1}^{(x)}$ for $\mathrm{x}$ on $\left.\mathrm{y}\right)$ and indirect influences from lag $h=t-1$ to $h=2$ on the mean structure of the other process. All of the indirect effects of one process on the other, from lag $h=t-1$ to $h=2$, come in the form of composites of autoregressive and cross-lagged paths. Namely, the mediated influence of one process on the other process via the other process (i.e., $\sum_{h=t-1}^{h=2}\left(\left[\rho_{x x}^{(h)} \rho_{x y}^{(h+1)}\right] \alpha_{t-h}^{(y)}\right)$ for y on x via x $\left.\& \sum_{h=t-1}^{h=2}\left(\left[\rho_{y y}^{(h)} \rho_{y x}^{(h+1)}\right] \alpha_{t-h}^{(x)}\right)\right)$ for x on y via y) and the mediated influence of one process on another process via itself (i.e., $\sum_{h=t-1}^{h=2}\left(\left[\rho_{y y}^{(h)} \rho_{x y}^{(h+1)}\right] \alpha_{t-h}^{(y)}\right)$ for y on x via y $\& \sum_{h=t-1}^{h=2}\left(\left[\rho_{x x}^{(h)} \rho_{y x}^{(h+1)}\right] \alpha_{t-h}^{(x)}\right)$ for x on y via x $)$.

In both configurations of CLPM, either fitting to residuals or to observed variables, the causal interpretation of CLPM suffers an omitted variable problem. The problem arises because CLPM only considers change in rank ordering amongst individuals over time, thereby confounding inter- and intra-individual effects with one another (Hamaker et al., 2015; Curran et al., 2014). In this regard, the omitted variable problem is due to unobserved inter-individual 
variability (Usami et al., 2019; Zyphur et al., 2019a, 2019b; Eichler, 2013). When exploring changes over time, omitted variables may be characterized as either stable (i.e., time-invariant) or changing over time (i.e., time-varying). Various models have been developed that account for such confounding, with some only accounting for stable, time-invariant differences in trajectory and others accounting for time-varying differences in change as well (see Usami et al., 2019; and, Zyphur et al. 2019b for an overview).

Fitting the lagged relations to the observed variables (eq. 2a-b) results in describing the between-person model in the same set of equations as the lagged relations, while fitting the lagged relations to the residuals (eq. 1c-d) results in modeling the lagged relations separately from the between-person model. On this point, when fitting to the observed variables the between-person factors may be interpreted as accumulating factors, and when fitting to the residuals the between-person factors can be interpreted as growth curve parameters (Usami et al., 2019). These interpretations are based on the way in which trajectories are captured, models fitting the lagged relations to the observed variables will incorporate both the between-person factors and the lagged relations in determining the trajectories, while fitting to the residuals results in the trajectories being determined by the between-person factors alone (i.e., as is done with growth curve models) ${ }^{1}$.

If we conceive of the overall trajectory as something which needs separated before considering the lagged relations, then we might opt to fit the lagged relations to the residuals. For example, say we have scores from standardized tests assessing students' math and science skills

\footnotetext{
${ }^{1}$ It should be noted that there is evidence that under certain conditions (viz., time-invariant autoregressive paths which do not exhibit a random walk $|A R|=1$ or explosive process $|A R|>1)$, models fitting lagged relations on the residuals are algebraically equivalent to models fitting lagged relations to the observed variables (Hamaker, 2005). This is specifically demonstrated between ALT (Curran \& Bollen, 2001) and the Latent Growth Curve with autocorrelated disturbances (Chi and Reinsel, 1989).
} 
from grade 1 to grade 8 and we want to investigate the grade to grade reciprocal relations between math and science skills. We may first wish to remove the overall trend reflecting the tendency for math and science skills to increase as students' progress through their studies before examining the lagged relations reflecting the grade to grade reciprocal effects between math and science skills. In this way, we are conceiving of the overall changes in math and science skills to be a separate process from, say, the effect of math skills on science skills when moving from $6^{\text {th }}$ to $7^{\text {th }}$ grade. On the other hand, if we wanted to consider overall change as working in concert with the lagged relations in describing the relations between processes over time, then we may opt to fit the lagged relations to the observed variables. In this way, the repeated measures on a construct serve as mediators explaining the influence of that construct's overall change on the repeated measures of another construct. For example, say we were to explore the reciprocal relationship between alcohol usage and anxiety over time. If we considered that increasing anxiety over time would manifest itself through increased occasion specific anxiety which would in turn lead to subsequent increases in occasion specific alcohol usage, then we would be considering this causal feedback process between anxiety and alcohol usage over time in the same dynamic system which incorporate both occasion to occasion elements as well as overall trajectories. Fitting the lagged relations to the observed variables allows the between-subject factors to have both direct and indirect effects on the observed indicators of the within-subject processes. In line with these substantive considerations, a motivation for fitting to the residuals has to do with distinguishability of the between-person trend and the within-person lagged relations, however under certain conditions (e.g., a random walk) the growth curve parameters will not be identifiable due to the conflation of deterministic and stochastic trends. 
Fitting lagged relations in the presence of between-person factors capturing overall change can lead to an issue of process distinguishability between the overall change (i.e., pertaining to the deterministic model) and lagged relations (i.e., pertaining to the stochastic model) (Clark et al., 2020, 2018; Jongerling \& Hamaker, 2011; Voelkle, 2008). Two related models, elaborated below, the autoregressive latent trajectory (ALT) model and the latent growth curve with structured residuals (LGCM-SR) model both incorporate a between-subject structure for change and stability along with lagged relations. However, the two models differ in terms of whether the lagged relations are fit to the residuals (i.e., LGCM-SR) or the observed variables (i.e., ALT). The motivation for developing the LGCM-SR was to provide a pure disaggregation of the between-subject structure from the lagged relations (Curran et al., 2014). Thus, it would follow that the decision to fit to the residuals may in part be influenced by the desire to provide better process distinguishability. Some preliminary evidence suggests that the LGCM-SR does provide better process distinguishability than the ALT, especially in the presence of a smaller number (<20) of time points that typically characterize panel data (Clark et al., 2020).

The identification of such models assumes that the between-person structure (i.e., the growth-curve) is capturing overall deterministic trends, such as the continuous upward growth of an individual's vocabulary in the process of acquiring linguistic competency. The deterministic trend pertains to how the expected value changes over time, which is the sort of detrending that allows for the process distinguishability discussed above. However, in time series we may also encounter stochastic trends, in which a process can exhibit unpredictable short-run behaviors (see Figure 1 below for an illustration of deterministic and stochastic trends). The problem of stochastic trends is that different individuals would be characterized by separate indeterminate patterns which the between-subject structure would try to account for, resulting in a highly 
convoluted interpretation for the between-subject growth components. Moreover, if specifying trends to exhibit particular form (e.g., linear), growth curve parameters may not be estimable at all, whereas if trends are allowed to be estimated from data (i.e., unspecified) then growth curve parameters may be estimable but would still have interpretive problems due to the conflation of deterministic and stochastic trends into "drift parameters".

Figure 1: Stochastic and Deterministic Trends for $10(t=10) \& 100$ time points ( $t=100)$ for two different, hypothetical individuals ( $i=A \& i=B$ ).
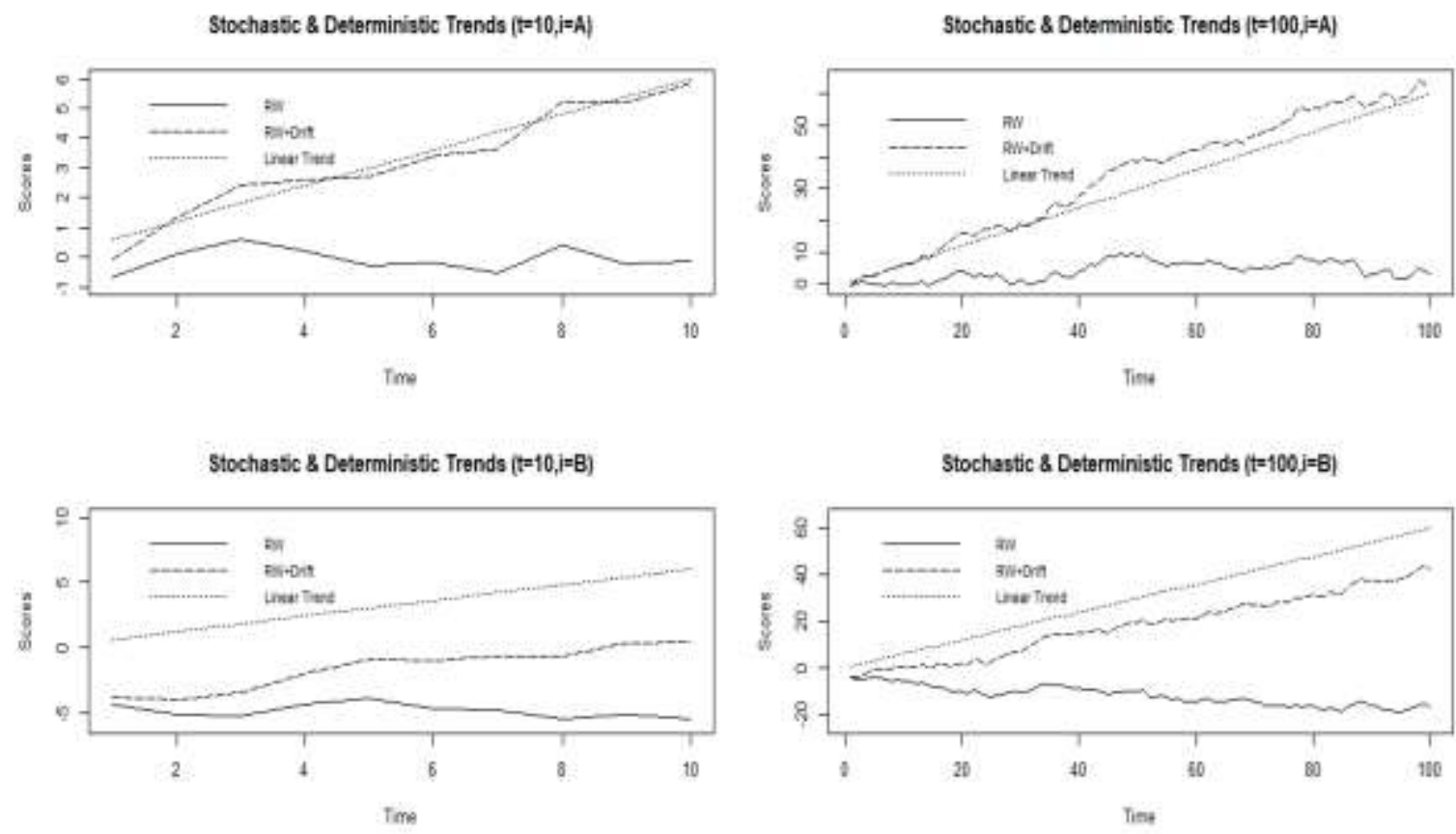

In time series we incorporate the lagged relations to account for intra-individual dependencies due to measures being close in time, and in so doing hope to reduce out any stochastic trends such that remaining unexplained error can be assumed as random noise. Within the AR terms, stochastic trends will persist in the presence of either a random walk (i.e., $|\mathrm{AR}|=1$ ) or an explosive process (i.e., $|A R|>1$ ), the former implies the influence of events at prior occasions on subsequent occasions will persist indefinitely into the future while the latter implies that the influence of events at prior occasions on subsequent occasions will become more and more 
amplified with each subsequent future occasion. These types of processes will lead to variance increasing as a function of time, i.e. variance non-stationarity. The growth-parameters are intended to deal with mean non-stationarity alone as would be exhibited by a deterministic trend. In the presence of a stochastic trend, the estimation of the growth-parameters will also be affected by this variance non-stationarity. Hence, the estimated growth-parameters will conflate the deterministic trend with the stochastic trend. Hence, in the presence of a stochastic trend, the process distinguishability discussed above would no longer hold. Further, when the stochastic trend incorporates drift (i.e., mean and variance non-stationarity), then the growth-parameters would be best described as drift parameters.

In this paper we will consider models that capture inter-individual differences in trajectories and lagged relations but differ in terms of (a) whether they account for time-variant differences in trajectory, and (b) whether they fit the lagged relations to the residuals or the observed variables. The models to be considered here are the random intercept cross-lagged panel model (RI-CLPM), the latent growth curve with structured residuals (LGCM-SR), the autoregressive latent trajectory model(ALT), and the General Cross-Lag Model (GCLM) with and without mean stationarity. These models will have specifications allowing the functional form of overall trajectory to take on any functional form (e.g., linear trends are not imposed).

These models will be illustrated with an empirical example and compared in the context of a simulation study. Specifically, we will compare amongst these models in terms of model fit and selection to get some sense of how we may go about choosing models to best match the underlying data generating process. To this end, we will consider commonly used fit indices and their respective, conventional criteria in determining whether a model fits data well. Model selection will be addressed from the perspective of using Bayes Factor as constructed from BIC, 
which provides a useful assessment that accounts for model fit in addition to model complexity and further allows for the comparison amongst both nested and non-nested models (Kass \& Raftery, 1995; Jeffreys, 1998; Raftery, 1999).

Of central interest in this study is the effect of ignoring time-varying inter-individual differences in trajectory on the estimation of cross-lagged paths. This interest is motivated by the emphasis researchers place on interpreting cross-lagged paths to reveal causal relations. Since inter-individual differences can be considered as confounders of these causal interpretations, the current study, through simulation, will be evaluating the extent to which failing to account for inter-individual differences in trends influences the cross-lagged path estimates and standard errors. To be exact, we will be generating from two different models that are characterized by inter-individual differences in trend (viz., LGCM-SR and GCLM) and fitting models which do not account for these differences in trend (viz., CLPM, Mean Stationary GCLM, and RI-CLPM). As an additional consideration we will fit models to data generated from CLPM which is not characterized by inter-individual differences in trajectory, and LGCM which is characterized only by inter-individual differences in trajectories. In the former case we will be able to assess the extent to which overfitting biases cross-lagged path estimates and standard errors; in the latter case, we can consider how models perform in terms of recovering the true lagged relations of 0 . In the following we detail the specifications for the different models under consideration in this study. Figure 2 depicts path diagrams for the models to be considered. 
(a) RI-CLPM

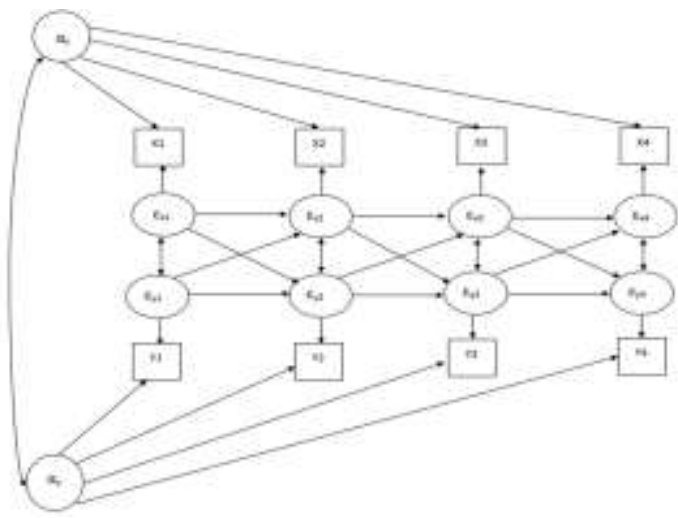

(c) Predetermined ALT (b) LGCM-SR

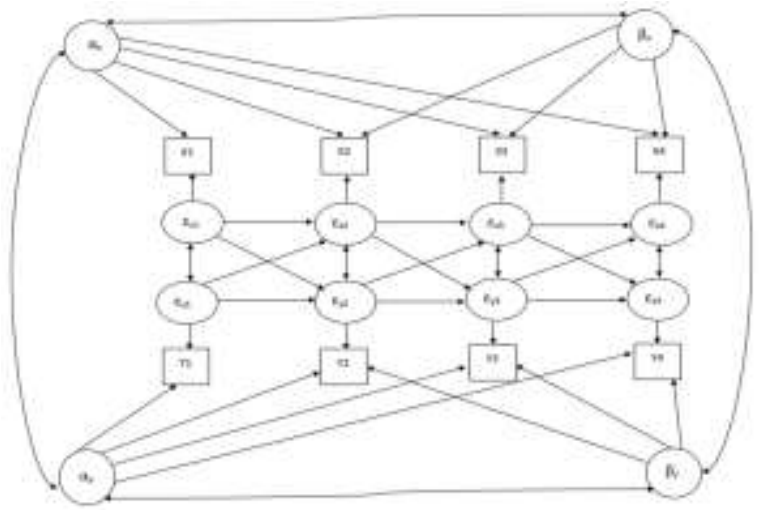

(d) GCLM

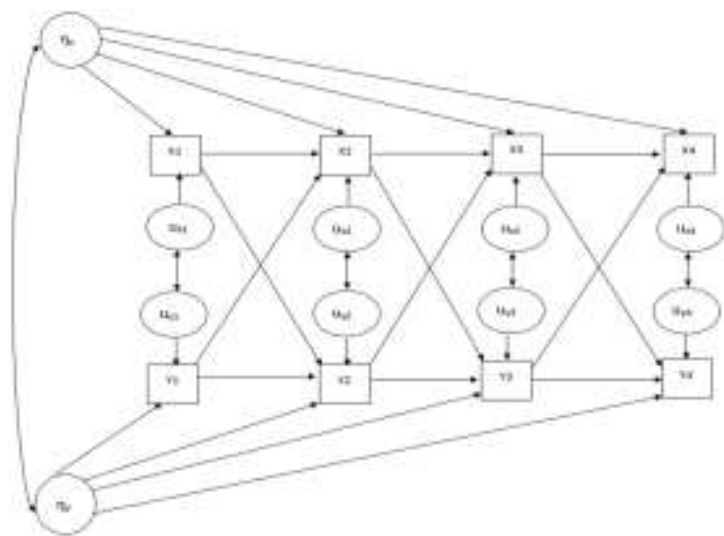

Figure 2. Path diagrams giving examples of the models considered in this study.

Panel (a) gives AR(1)CL(1) RI-CLPM (Note: All paths from Latent Variables for Random Intercept and Structured Residuals to Observed Variables are constrained to 1 with CLPM described in eq. 1a-d); Panel (b) gives $A R(1) C L(1) L G C M$-SR (Note: covariance paths across process intercepts and slopes omitted for simplicity; the paths from the Latent Variables for Random Intercept and Structured Residuals to the Observed Variables are set at 1, while Random Slope loadings at set to 1 at time 2 and freely estimated at all subsequent measures); Panel (c) gives $A R(1) C L(1)$ predetermined ALT (Note: the loadings at time 2 are constrained to 1, with all others being freely estimated); Panel (d) gives AR(1)CL(1) GCLM (Note: when Mean Stationarity holds the paths from the Latent Variables for Unit Effects to the Observed Variables will be fixed to 1 (excepting one loading to be freely estimated), otherwise we allow all but one loading to be freely estimated). 
Random Intercept-Cross Lagged Panel Model (RI-CLPM)

Hamaker et al. (2015) proposed the Random Intercept Cross-lagged Panel Model (RI-CLPM) to account for confounding due to trait-like stability by incorporating a random intercept into the CLPM specification given in equations 1a-d. Further, by allowing intercepts to covary, one can also capture the overall stable relationship between constructs at the between-person level:

$$
\begin{gathered}
y_{i t}=\mu_{t}^{(y)}+\delta_{i}^{(y)}+\epsilon_{i t}^{(y)} \\
x_{i t}=\mu_{t}^{(x)}+\delta_{i}^{(x)}+\epsilon_{i t}^{(x)}
\end{gathered}
$$

The random intercepts, $\delta_{i}$, are incorporated into the mean structure as variance terms to capture individuals' deviations from the group level means over time, $\mu_{t}$, allowing each individual to have their trajectory over time to be distinct from the group-level trajectory. However, this distinction between the group-level trajectory and the individual's specific deviation from that group level trajectory will be constant. For example, perhaps we find that students' anxiety levels over a semester exhibit a cubic trend such that anxiety goes up towards mid-terms then subsequently down and then up again approaching finals. Moreover, we also know that some students tend to be higher or lower on anxiety in general, thus we may anticipate that they too will exhibit this cubic trend, with the distinction that the higher anxiety students show a parallel trend situated above the group-level trend and the lower anxiety students show a parallel trend situated below the group-level trend. The overall group-level trend will be captured in the time-specific means, $\mu_{t}$, while the individual's distinct, parallel trends will be captured in the individual-specific deviations, $\delta_{i}$. Figure 2a gives a path diagram for the RI-CLPM. 
Latent Growth Curve Model with Structured Residuals (LGCM-SR)

Curran et al. (2014) proposed the Latent Growth Curve Model with Structured Residuals (LGCM-SR). This model utilizes a Latent Growth Curve to detrend variables before considering the CLPM as given in equations 1a-d. This model is distinguished from the RI-CLPM by allowing one to evaluate the CLPM after accounting for both overall inter-individual differences in standing on a construct as well as inter-individual differences in overall change on a construct across time. Though it is common to specify the functional form of growth as linear, this is not essential. One may also allow the trajectory to be freely estimated by leaving slope loadings unspecified (Meredith \& Tisak, 1990). The LGCM-SR with an unspecified growth trajectory (LGCM-SR-UGT) requires that one set two slope loadings to identify the intercept $(\lambda=0)$ and a reference for the rate of change $(\lambda=1)^{2}$.

The addition of the model parameters for capturing inter-individual differences in change over time (i.e., random slope terms) establishes a between-person structure containing grouplevel trajectories (i.e., fixed intercept and slope parameters) and the corresponding individual specific deviation terms (i.e., random effects for intercept and slope) along with an unstructured covariance pattern for the random effects:

$$
\begin{gathered}
y_{i t}=\alpha_{i}^{(y)}+\lambda_{t} \beta_{i}^{(y)}+\epsilon_{i t}^{(y)} \\
x_{i t}=\alpha_{i}^{(x)}+\lambda_{t} \beta_{i}^{(x)}+\epsilon_{i t}^{(x)}
\end{gathered}
$$

With covariances amongst the exogenous variables $\left(\alpha_{i}^{(x)}, \alpha_{i}^{(y)}, \beta_{i}^{(x)}, \beta_{i}^{(y)}\right)$ being freely estimated. Where $\alpha_{i}^{(y)} \& \alpha_{i}^{(x)}$ represent the intercept terms with fixed, group-level means,

\footnotetext{
${ }^{2}$ It should be noted that technically, in theory, any two values can be used for identification allowing the remaining loadings to be freely estimated (McArdle \& Hamagami, 1991).
} 
$\mu_{\alpha}^{(y)} \& \mu_{\alpha}^{(x)}$ plus individual deviations from the overall group-level intercept $\delta_{i}^{(y)} \& \delta_{i}^{(x)}$, allowing $\mu_{\alpha}^{(y)}+\delta_{i}^{(y)} \& \mu_{\alpha}^{(x)}+\delta_{i}^{(x)}$ to capture overall stable differences amongst individuals. The exact interpretation of these intercept terms will depend on the way in which we specify them. A common approach is to interpret the intercept relative to the initial time point, effectively allowing us to control out pre-existing differences amongst individuals which would be expected to persist over time. For example, individual's with generally higher levels of anxiety could be expected to enter the term at a higher level of anxiety than most others, then over the course of the term may be expected to persistently have higher anxiety (i.e., this is the kind of trajectory captured in RI-CLPM). However, what if this student showed less change in anxiety over the term than others, or perhaps, opposite of other students, even showed a decline in anxiety approaching mid-term. To account for such possibilities the LGCM-SR fits fixed effects for group-level mean rates of change over time, $\mu_{\beta}^{(y)}$ and $\mu_{\beta}^{(x)}$ along with time specific rates of change, $\lambda_{t}^{(y)}$ and $\lambda_{t}^{(x)}$, capturing the functional form of the group-level trajectory over time. The individual specific deviations from the group-level average change over time, $\zeta_{i}^{(y)}$ and $\zeta_{i}^{(x)}$, along with the corresponding interactions with the time-specific rates of change provides the possibility for individuals' to be characterized by their own trajectory over time via $\lambda_{t}^{(y)}\left(\mu_{\beta}^{(y)}+\zeta_{i}^{(y)}\right)$ and $\lambda_{t}^{(x)}\left(\mu_{\beta}^{(x)}+\zeta_{i}^{(x)}\right)$. Figure $2 \mathrm{~b}$ provides a path diagram giving an example of the LGCM-SR.

We can establish LGCM-SR-UGT by setting time point 1 slope loading at $\lambda=0$ to capture time point 1 mean in the intercept and time point 2 at $\lambda=1$ to establish a base rate of change $\left(\mu_{\beta}=\mu_{2}-\mu_{1}\right)$ then leave the remaining elements in $\lambda$ unconstrained so that they may be freely estimated. This allows a growth trajectory that reflects the mean structure of a construct over time in terms of relative rates of change. In other words, when the first two loadings are set at 0 
$\& 1$, the growth trajectory estimates a modified mean spline trajectory (Preacher et al., 2008;

Hancock \& Lawrence, 2006). For time points $\mathrm{t}=\{1, \ldots, \mathrm{s}\}$ this can be expressed as:

$$
\hat{\lambda}=\left(\begin{array}{c}
0 \\
1 \\
\frac{\hat{\mu}_{3}-\hat{\mu}_{1}}{\hat{\mu}_{2}-\hat{\mu}_{1}} \\
\vdots \\
\frac{\hat{\mu}_{s}-\hat{\mu}_{1}}{\hat{\mu}_{2}-\hat{\mu}_{1}}
\end{array}\right)
$$

Giving slope loading parameters a generic model form of:

$$
\lambda_{t}=\frac{\mu_{t}-\mu_{1}}{\mu_{2}-\mu_{1}}
$$

The model for this LGCM with an unspecified growth trajectory (LGCM-UGT) can then be given for a process as:

$$
y_{i t}=\alpha_{i}+\frac{\mu_{t}-\mu_{1}}{\mu_{2}-\mu_{1}} \beta_{i}+\varepsilon_{i t}
$$

Where $\alpha_{i}=\mu_{1}+\delta_{i}$ gives a random intercept term for each $i^{\text {th }}$ individual having time point 1 mean $\mu_{1}$ with random effect $\delta_{i}$; and, $\beta_{i}=\mu_{\beta}+\zeta_{i}$ gives a random slope for each $i^{\text {th }}$ individual with mean base rate of change $\mu_{\beta}$ and random effect $\zeta_{i}$. Slope loadings are interpreted relative to the base rate of change $\mu_{\beta}=\mu_{2}-\mu_{1}$; and, $\varepsilon_{i t}=y_{i t}-\left(\alpha_{i}+\lambda_{t} \beta_{i}\right)$ capturing the time specific deviation of individual $i$ at time point $t$. Fitting the slope terms changes the substantive interpretation of the intercept, namely LGCM-SR-UGT as specified above gives the intercept as time point 1 standing while the RI-CLPM has the intercept capturing trait-like stability over time. RI-CLPM can be understood as nested in LGCM-SR-UGT. 


\section{Relating RI-CLPM to LGCM-SR-UGT}

The RI-CLPM can be cast as nested in the LGCM-SR-UGT by reparameterizing the RICLPM to estimate the mean structures through the trajectory as is done with the LGCM-SR-UGT.

The measurement model for the RI-CLPM can be given as:

$$
y_{i t}=\delta_{i}+\mu_{t}+\varepsilon_{i t}
$$

Where $\delta_{i}$ captures the person-specific trait-like stability, i.e. random intercept effect.

If the Slope Variances for the LGCM-SR-UGT are set to zero, then we get the measurement model:

$$
y_{i t}=\alpha_{i}+\frac{\mu_{t}-\mu_{1}}{\mu_{2}-\mu_{1}} \mu_{\beta}+\varepsilon_{i t}
$$

Which provides an alternative parameterization of the RI-CLPM such that:

$$
\begin{gathered}
\alpha_{i}+\frac{\mu_{t}-\mu_{1}}{\mu_{2}-\mu_{1}}\left(\mu_{2}-\mu_{1}\right)+\varepsilon_{i t}= \\
\mu_{1}+\delta_{i} \frac{\mu_{t}-\mu_{1}}{\mu_{2}-\mu_{1}}\left(\mu_{2}-\mu_{1}\right)-\mu_{1}+\varepsilon_{i t}= \\
\mu_{1}+\frac{\mu_{t}-\mu_{1}}{\mu_{2}-\mu_{1}}\left(\frac{\mu_{2}-\mu_{1}}{1}\right)+\delta_{i}+\varepsilon_{i t}= \\
\mu_{1}+\frac{\mu_{t}-\mu_{1}\left(\mu_{2}-\mu_{1}\right)}{\mu_{2}-\mu_{1}}+\delta_{i}+\varepsilon_{i t}= \\
\mu_{1}+\mu_{t}-\mu_{1}+\delta_{i}+\varepsilon_{i t}= \\
\mu_{t}+\delta_{i}+\varepsilon_{i t}
\end{gathered}
$$


Showing how we can derive the RI-CLPM measurement model from the LGCM-SR-UGT which estimates a modified mean spline and has zero slope variance. Substituting in the time-specific means as $\mu_{t}=\mu_{1}+\lambda_{t} \mu_{\beta}$ gives the reparameterized version of the RI-CLPM:

$$
y_{i t}=\mu_{1}+\lambda_{t} \mu_{\beta}+\delta_{i}+\varepsilon_{i t}
$$

Given that the CLPM specification for the RI-CLPM and LGCM-SR-UGT are both represented by equations $1 \mathrm{c}-\mathrm{d}$, then we can express the models as:

$$
\begin{array}{ll}
\text { LGCM-SR-UGT: } & y_{i t}=\mu_{1}+\lambda_{t}\left(\mu_{\beta}+\zeta_{i}\right)+\delta_{i}+\varepsilon_{i t}^{*} \\
\text { RI-CLPM: } & y_{i t}=\mu_{1}+\lambda_{t} \mu_{\beta}+\delta_{i}+\varepsilon_{i t}^{*}
\end{array}
$$

Indicating that the RI-CLPM is a reduced form of LGCM-SR-UGT where the slope deviations, $\zeta_{i}$, and the associated covariances are excluded, thus when considering two processes, RI-CLPM will have seven fewer parameter estimates than LGCM-SR-UGT. Within this framework, we can assess if accounting for inter-individual differences in trajectory would improve model fit by performing a model comparison between an LGCM-SR-UGT with non-zero slope variance to an LGCM-SR-UGT with slope variance set to zero.

Autoregressive Latent Trajectory Model (ALT)

The Autoregressive Latent Trajectory (Bollen \& Curran, 2004; Bollen \& Zimmer, 2010) model is closely related to the LGCM-SR in the sense that it fits a Latent Growth Curve (LGCM) to account for between-person differences in standing and changes over time, however ALT fits the CLPM as in equations $2 \mathrm{a}-\mathrm{b}$. Instead of capturing the mean structure by using occasion effects (i.e., time-specific intercepts, $\alpha_{t}^{(x)} \& \alpha_{t}^{(y)}$ ) in conjunction with the AR \& CL 
paths, the ALT zeroes-out these occasion effects and uses "growth-curve" parameters ${ }^{3}$ in conjunction with the AR \& CL paths.

There is a known issue with ALT concerning the initial condition specifications (Bollen \& Curran, 2004; Hamaker, 2005; Jongerling \& Hamaker, 2011; Ou et al., 2017; Allison et al., 2017) and the "starting up" of the AR process. To handle this issue, ALT can be given a predetermined specification wherein the variables at the first measurement occasion are treated as exogenous by allowing them to covary with the other exogenous variables in the model (i.e., accumulating factors). Exogenous observed variables at time point one take on a similar role as the random intercept terms of the LGCM-SR-UGT presented above where the intercept is interpreted relative to the initial time point. The accumulating factors of the ALT may be left unspecified allowing the trajectory to be freely estimated. The predetermined ALT with a freely estimated trajectory will account for inter-individual differences in overall standing in the variance of the observed variables at time point 1 , while inter-individual difference in overall change will be captured in the accumulating factor for the trajectory. This predetermined ALT with unstructured trajectory can be specified as such:

$$
\begin{aligned}
& y_{i t}=\lambda_{t} \beta_{i}^{(y)}+\rho_{y y} y_{i t-1}+\rho_{y x} x_{i t-1}+u_{i t}^{(y)} \\
& x_{i t}=\lambda_{t} \beta_{i}^{(x)}+\rho_{x x} x_{i t-1}+\rho_{x y} y_{i t-1}+u_{i t}^{(x)}
\end{aligned}
$$

With covariances amongst the exogenous variables $\left(\beta_{i}^{(y)}, \beta_{i}^{(x)}, y_{i 1}, \& x_{i 1}\right)$ freely estimated. The between person components, $\beta_{i}^{(y)}=\mu_{\beta}^{(y)}+\zeta_{i}^{(y)} \& \beta_{i}^{(x)}=\mu_{\beta}^{(x)}+\zeta_{i}^{(x)}$, as before have a fixed

\footnotetext{
${ }^{3}$ As Usami et al. (2019) have aptly pointed out, these should rather be considered as accumulating factors since they work in concert with the AR \& CL paths to establish the change over time.
} 
mean rate of change term, $\mu_{\beta}$, and individual specific deviations for rate of change, $\zeta_{i}$, capturing time-varying overall inter-individual differences; while $x_{i 1}=\mu_{x_{1}}+\epsilon_{i 1}^{(x)} \& y_{i 1}=\mu_{y_{1}}+\epsilon_{i 1}^{(y)}$, have group-level mean at the initial time point, $\mu_{1}$, and individual specific deviations about the initial time point, $\epsilon_{i 1}$, capturing individuals overall pre-existing differences. This ALT has the group-level mean trajectory for the CLPM represented as:

$$
\begin{aligned}
& \mu_{t}^{(x)}=\lambda_{t} \mu_{\beta^{(x)}}+\rho_{x x} \mu_{t-1}^{(x)}+\rho_{x y} \mu_{t-1}^{(y)} \\
& \mu_{t}^{(y)}=\lambda_{t} \mu_{\beta^{(y)}}+\rho_{y y} \mu_{t-1}^{(y)}+\rho_{y x} \mu_{t-1}^{(x)}
\end{aligned}
$$

Interactions of the random effects terms $\zeta_{i}^{(x)} \& \zeta_{i}^{(y)}$ with time specific rates of change loadings $\lambda_{t}$ allow individuals to take on their own trajectory, while an individual's mean trajectory is started up by $\mu_{i 1}+\epsilon_{i 1}$, where the initial time point is treated as an exogenous variable ${ }^{4}$. Figure $2 \mathrm{c}$ depicts a path diagram for predetermined ALT.

\section{General Cross-lagged Model}

The General Cross-lagged Panel Model (GCLM) was introduced by Zyphur et al. (2019a) to provide a framework to account for time-varying and time-invariant effects at the betweenperson level and extend the lagged dynamics over time in order to bolster our ability to capture causal effects from panel data. GCLM controls for inter-individual differences in trajectory through specifications of "unit effects". To be consistent with the other models in this paper, we will consider GCLM specified with an AR(1)CL(1) CLPM as given in equations 2a-b. Figure 2d gives a generic depiction of this model.

\footnotetext{
${ }^{4}$ Setting trajectory variance $\zeta_{i}=0$ reduces this ALT to a reparametrized version of the CLPM as given in equations 2a-b with $\alpha_{t}^{(x)}=0 \& \alpha_{t}^{(y)}=0$, and the mean structure as given in equations $15 \mathrm{a}-\mathrm{b}$.
} 
The unit effects of the GCLM are primarily intended to account for overall betweenperson differences in standing over time such that the CLPM may be interpreted in terms of causal relations amongst processes as they change over time. These stable between person differences will be represented as $\eta_{i}^{(x)} \& \eta_{i}^{(y)}$. As with other models discussed above, these unit effects allow an individual to have a long-run deviation from the group-level mean trajectory. The covariance of unit effects are freely estimated to allow relations between processes at the person level to be controlled for when assessing the causal effects implied by the CLPM. If we wish only to account for stable inter-individual differences over time (i.e., time-invariant unit effects), then we can specify the unit effects as exhibiting mean stationarity, this is what we will refer to as the Mean Stationary GCLM, which can be formulated as such:

$$
\begin{aligned}
& y_{i t}=\alpha_{t}^{(y)}+\eta_{i}^{(y)}+\rho_{y y} y_{i t-1}+\rho_{y x} x_{i t-1}+u_{i t}^{(y)} \\
& x_{i t}=\alpha_{t}^{(x)}+\eta_{i}^{(x)}+\rho_{x x} x_{i t-1}+\rho_{x y} y_{i t-1}+u_{i t}^{(x)}
\end{aligned}
$$

These models are established by fixing all but one of the loadings from the unit effects to the time specific measures to 1 , each process must have the loadings at time point 1 freely estimated $\left(\lambda_{1} \eta_{i}\right)$ for the purposes of identifying the unit effects as capturing the adjusted overall mean scores of individuals accounting for lagged relations across time.

In this formulation, we see that an individual's deviation from the overall group-mean is held constant over time (i.e., this is what is meant by mean stationarity), which controls for overall between-person stability across time. However, if we wanted to allow individuals' deviations from the overall group-mean to vary at different time points, then we would need to incorporate time-varying unit effects. This is accomplished by allowing the loadings of timespecific measures on the unit effects to be freely estimated, simply referred to as GCLM: 


$$
\begin{aligned}
& y_{i t}=\alpha_{t}^{(y)}+\lambda_{t}^{(y)} \eta_{i}^{(y)}+\rho_{y y} y_{i t-1}+\rho_{y x} x_{i t-1}+u_{i t}^{(y)} \\
& x_{i t}=\alpha_{t}^{(x)}+\eta_{i}^{(x)}+\rho_{x x} x_{i t-1}+\rho_{x y} y_{i t-1}+u_{i t}^{(x)}
\end{aligned}
$$

To identify the unit effects with an observed variable scale, the loading for one of the time points must be fixed to one, in line with Zyphur et al. (2019a) we chose the last time point loading. By fitting the freely estimated loadings (i.e., time-varying unit effect), the influence of an individuals' overall standing (i.e., time-invariant unit effect) is moderated by time-specific effects at the between-person level. The GCLM without mean stationarity is in line with the LGCM-SR and ALT in that it allows for time-varying differences at the between-person level, while the Mean Stationary GCLM is more in line with the RI-CLPM in that both only control for time-invariant differences amongst individuals.

\section{Empirical Example}

In the following we fit each of these models to data from the Longitudinal Study of American Youth (LSAY 1987-1994). The models are fit to examine the association between annual IRT scores for Math and Science Achievement from grades 7 to 12 with a sample of n=3109 students using MPlus 8.4 (Muthén \& Muthén,1998-2017) with full information maximum likelihood estimation. For simplicity we constrain the AR and CL paths to be equal over time, we also impose equality amongst the time-specific residual covariances (aka comovements) over time ${ }^{5}$.

\footnotetext{
${ }^{5}$ Note: for the predetermined ALT, the covariance at time point 1 are considered exogenous hence they are not constrained to be equal with the covariance at subsequent time points which are considered endogenous.
} 
Table 1: Results from fitting different models to LSAY data example.

\begin{tabular}{|c|c|c|c|c|c|}
\hline Models & $R I-C L P M$ & $L G C M-S R-U G T$ & $\begin{array}{r}\text { Predetermined } \\
A L T \\
\end{array}$ & $\begin{array}{r}\text { Mean Stationary } \\
\text { GCLM } \\
\end{array}$ & GCLM \\
\hline Within-Person CLPM Parameters: & Estimate $(S E)$ & Estimate (SE) & Estimate (SE) & Estimate (SE) & Estimate (SE) \\
\hline AR Math & $0.69(0.014)$ & $0.316(0.028)$ & $0.404(0.019)$ & $0.538(0.023)$ & $0.396(0.020)$ \\
\hline AR Science & $0.60(0.017)$ & $0.354(0.026)$ & $0.393(0.019)$ & $0.463(0.024)$ & $0.417(0.020)$ \\
\hline CL Math $\rightarrow$ Science & $0.261(0.013)$ & $0.049(0.020)$ & $0.091(0.016)$ & $0.084(0.012)$ & $0.040(0.012)$ \\
\hline CL Science $\rightarrow$ Math & $0.265(0.013)$ & $0.046(0.020)$ & $0.087(0.016)$ & $0.073(0.013)$ & $0.045(0.013)$ \\
\hline Math $\leftarrow \rightarrow$ Science (time-specific) & $10.735(0.40)$ & $5.122(0.460)$ & $6.099(0.407)$ & $7.753(0.378)$ & $6.742(0.345)$ \\
\hline \multicolumn{6}{|l|}{$\begin{array}{l}\text { Range of Residual Variances across } \\
\text { Time }\{s=1 \text { to } 6\} \text { : }\end{array}$} \\
\hline Math & $\begin{array}{l}21.658- \\
34.048 \\
(0.927-1.836) \\
\end{array}$ & $\begin{array}{r}22.388-25.145 \\
(0.931-1.809)\end{array}$ & $\begin{array}{l}24.912-26.353 \\
(0.909-1.300)\end{array}$ & $\begin{array}{l}24.543-30.787 \\
(0.889-1.311)\end{array}$ & $\begin{array}{l}24.589-29.312 \\
(0.910-1.307)\end{array}$ \\
\hline Science & $\begin{array}{l}23.261- \\
38.424 \\
(0.880-1.692) \\
\end{array}$ & $\begin{array}{l}15.682-30.955 \\
(0.914-2.569)\end{array}$ & $\begin{array}{l}17.469-29.256 \\
(0.843-1.191)\end{array}$ & $\begin{array}{l}18.086-34.744 \\
(0.803-1.251)\end{array}$ & $\begin{array}{l}18.100-33.748 \\
(0.878-1.194)\end{array}$ \\
\hline \multicolumn{6}{|l|}{$\begin{array}{l}\text { Time Specific Intercepts/ Occasion } \\
\text { Effects }\{s=1 \text { to } 6\}:\end{array}$} \\
\hline Math & $\begin{array}{l}\{50.348 \\
(0.185), \\
53.702(0.213), \\
57.831(0.233), \\
62.123(0.250), \\
64.877(0.269), \\
65.629(0.296)\}\end{array}$ & --- & --- & $\begin{array}{l}\{50.352(0.182), \\
26.128(0.564), \\
38.762(0.602), \\
41.626(0.648), \\
42.909(0.692), \\
42.255(0.723)\}\end{array}$ & $\begin{array}{l}\{50.352(0.182), \\
31.448(1.140), \\
34.057(1.219), \\
36.504(1.311), \\
37.471(1.397), \\
36.734(1.458)\}\end{array}$ \\
\hline Science & $\begin{array}{l}50.350 \\
(0.187), \\
53.667(0.206), \\
57.811(0.213) \\
59.997(0.225), \\
62.003 \\
(0.236), \\
62.979(0.265)\} \\
\end{array}$ & $\begin{array}{ll}-- \\
\end{array}$ & --- & $\begin{array}{l}50.358(0.175), \\
38.292(0.578), \\
41.457(0.614), \\
42.382(0.663), \\
43.773(0.695), \\
43.805(0.728)\}\end{array}$ & $\begin{array}{l}\text { \{50.352(0.182), } \\
30.608(1.162), \\
33.267(1.239), \\
33.510(1.334), \\
34.327(1.397), \\
34.199(1.453)\}\end{array}$ \\
\hline \multicolumn{6}{|l|}{$\begin{array}{l}\text { Between-Person Component } \\
\text { Variances-Covariances: }\end{array}$} \\
\hline $\begin{array}{l}\text { Math Unit } \\
\text { Effect/Random Intercept }\end{array}$ & $84.056(3.023)$ & $79.342(2.999)$ & $103.818(2.642)$ & $64.082(2.306)$ & $75.028(6.134)$ \\
\hline $\begin{array}{l}\text { Science Unit } \\
\text { Effect/Random Intercept }\end{array}$ & $69.733(2.592)$ & $73.061(3.456)$ & $104.286(2.656)$ & $59.731(2.222)$ & $51.650(4.803)$ \\
\hline $\begin{array}{l}\text { Math Accumulating } \\
\text { Factor (AF)/Random Slope }\end{array}$ & --- & $2.146(0.243)$ & $36.742(2.954)$ & --- & --- \\
\hline \begin{tabular}{l}
\multicolumn{1}{c}{ Science } \\
Accumulating Factor (AF)/Random \\
Slope
\end{tabular} & --- & $1.842(0.335)$ & $29.903(2.579)$ & --- & --- \\
\hline $\begin{array}{l}\text { Math-Science Intercepts/Unit } \\
\text { Effects }\end{array}$ & $66.143(2.408)$ & $69.438(2.326)$ & $74.537(2.301)$ & $23.350(2.493)$ & $55.193(4.139)$ \\
\hline Math Intercept-Math Slope/AF & --- & $8.044(0.606)$ & $50.601(2.514)$ & --- & --- \\
\hline Science Intercept- Science Slope/AF & --- & $6.079(0.833)$ & $44.067(2.343)$ & $\begin{array}{l}-- \\
\end{array}$ & --- \\
\hline Math Intercept-Science Slope/AF & --- & $5.127(0.551)$ & $39.095(2.296)$ & --- & --- \\
\hline Science Intercept-Math Slope/AF & --- & $7.022(0.512)$ & $42.889(2.362)$ & --- & 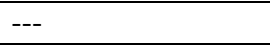 \\
\hline Math-Science Slopes/AFs & --- & $1.611(0.167)$ & $27.230(2.310)$ & --- & --- \\
\hline \multicolumn{6}{|l|}{ Between-Person Component Means: } \\
\hline Math Intercept & --- & $50.346(0.183)$ & $50.351(0.183)$ & --- & $\begin{array}{ll}-- \\
-\end{array}$ \\
\hline Math Slope/AF & --- & $3.288(0.114)$ & $28.826(1.194)$ & --- & --- \\
\hline Science Intercept & $\begin{array}{ll}-- \\
\end{array}$ & $50.360(0.183)$ & $50.353(0.184)$ & --- & $\begin{array}{ll}-- \\
\end{array}$ \\
\hline
\end{tabular}




\begin{tabular}{|l|l|l|l|l|l|}
\hline Science Slope/AF & --- & $3.341(0.122)$ & $29.242(1.180)$ & --- & -- \\
\hline $\begin{array}{l}\text { Slopel AF/ Unit Effect Loadings } \\
\text { \{s=1 to 6\}: }\end{array}$ & & & & & \\
\hline Math & --- & $\{0,1$, & $\{0,1$, & $\{1.637(0.102)$, & $\{0.986(0.040)$, \\
& & $2.302(0.066)$, & $1.093(0.005)$, & $1,1,1,1,1\}$ & $0.660(0.019)$, \\
& & $3.547(0.110)$, & $1.169(0.006)$, & & $0.855(0.021)$, \\
& $4.311(0.137)$, & $1.196(0.006)$, & & $0.882(0.022)$, \\
& & $4.593(0.148)\}$ & $1.183(0.008)\}$ & & $0.884(0.024), 1\}$ \\
\hline \multicolumn{1}{|c|}{ Science } & --- & $\{0,1$, & $\{0,1$, & $\{1.634(0.102)$, & $\{1.154(0.054)$, \\
& & $2.195(0.066)$, & $1.085(0.005)$, & $1,1,1,1,1\}$ & $0.777(0.025)$, \\
& & $2.842(0.093)$, & $1.092(0.006)$, & & $0.790(0.025)$, \\
& & $3.459(0.115)$, & $1.120(0.007)$, & & $0.846(0.026)$, \\
\hline Model Fit & & & & & $0.932(0.028), 1\}$ \\
\hline GOF Chi-Squared (df) & & & & \\
\hline CFI & $609.063(58)$ & $206.354(51)$ & $267.500(53)$ & $630.832(56)$ & $225.931(48)$ \\
\hline RMSEA (90\% CI) & 0.983 & 0.995 & 0.993 & 0.982 & 0.994 \\
\hline SRMR & 0.055 & $0.031(0.027$, & $0.036(0.032$, & $0.057(0.053$, & $0.035(0.030$, \\
\hline BIC & $0.051,0.059)$ & $0.036)$ & $0.040)$ & $0.062)$ & $0.039)$ \\
\hline & 0.112 & 0.044 & 0.061 & 0.087 & 0.016 \\
\hline
\end{tabular}

Generally, model fit indices suggest that these models have good fit using standard criteria (Hu \& Bentler,1999). We also see that though cross-lagged paths from the different models vary in magnitude, every model indicates that the paths from one process to the other are nearly equal, implying a reciprocating effect where neither process appears to causally dominate the other. Models accounting for variability in trajectory tend to yield smaller AR \& CL path estimates, with the exception that ALT yields similar estimates as the Mean Stationary GCLM. This may imply that failing to disentangle overall variability in change may lead to confounding relationships at the between-person level with lagged relations at the within-person level. Across models, correlations amongst the between-person components for math \& science achievement are considerably higher than the occasion specific and lagged relations suggesting that a students' overall standing and trajectory of math and science achievement across time may be more important than their grade to grade standings.

Given that the RI-CLPM is nested in the LGCM-SR-UGT we can use a chi-squared difference test to assess for significant model improvement, $\Delta \chi^{2}(7)=402.709, \mathrm{p}<0.001$. In this 
case we find that the model accounting for inter-individual variability in change over time significantly improves over a model which only accounts for inter-individual variability in overall standing. Similarly, Bayes Factor (as estimated by $B F_{i j}=e^{B I C_{i}-B I C_{j}}$ ) indicates that there is very strong evidence favoring the LGCM-SR-UGT over the RI-CLPM. See Table 2 for interpretive criteria for Bayes Factor (Kass \& Raftery, 1995; Jeffreys, 1998; Raftery, 1999).

Table 2. Interpretive Criteria for using Bayes Factor in Selecting amongst Models.

\begin{tabular}{|c|l|}
\hline Bayes Factor (BFij) Interpretation & \\
\hline $\mathrm{BFij}<=0.007$ & Very Strong Evidence for Model $\mathrm{i}$ \\
\hline $0.007<\mathrm{BFij}<=0.05$ & Strong Evidence for Model $\mathrm{i}$ \\
\hline $0.05<\mathrm{BFij}<=0.333$ & Moderate Evidence favoring Model $\mathrm{i}$ \\
\hline $0.333<\mathrm{BFij}<1$ & Weak Evidence for Model $\mathrm{i}$ \\
\hline $\mathrm{BFij}=1$ & No difference \\
\hline $1<\mathrm{BFij}<=3$ & Weak Evidence for Model $\mathrm{j}$ \\
\hline $3<\mathrm{BFij}<=20$ & Moderate Evidence favoring Model $\mathrm{j}$ \\
\hline $20<\mathrm{BFij}<=150$ & Strong Evidence for Model $\mathrm{j}$ \\
\hline $\mathrm{BFij}>150$ & Very Strong Evidence for Model $\mathrm{j}$ \\
\hline
\end{tabular}

Bayes Factor indicates that ALT is very strongly favored over RI-CLPM, once again indicating the importance of accounting for inter-individual differences in change. However, LGCM-SR-UGT is strongly favored over ALT, suggesting perhaps that separating the trends from the CLPM using the growth-curves may better represent the relations amongst math and science than combining the CLPM with the accumulating factors as a general process relating math and science. This may stem from the fact that most of the relationship between math and science over time appears to be accounted for at the between-person level. Since the Mean Stationary GCLM is nested under the GCLM a Chi-Squared difference test can be used for model comparison, $\Delta \chi^{2}(8)=404.901, p<0.001$, suggesting significant model improvement by allowing for time-varying unit effects. Bayes Factor suggests that RI-CLPM is very strongly favored over Mean Stationary GCLM. Similar to findings comparing ALT and LGCM-SR, Bayes Factor suggest that the model fit of LGCM-SR is very strongly preferred over that of 
GCLM, while there is only moderate evidence suggesting that GCLM is preferred over ALT $\left(B F_{i j}=0.257\right)$.

\section{Simulation Study}

The simulation study aims to evaluate (1) how do different models empirically relate in terms of model fit; and (2) what is the effect of ignoring time-varying inter-individual differences over time on cross-lagged parameter estimates. Data generation and analyses are conducted through MPlus 8.3 (Muthén \& Muthén, 1998-2017) and R 3.6 (R Core Team, 2019) using the MASS package (Venables \& Ripley, 2002), tidyverse functionality ( Wickham, 2017; Lee et al., 2020), and the MplusAutomation package (Hallquist \& Wiley, 2018).

\section{Generating Models and Conditions}

The primary generating models of interest are LGCM-SR with modified mean spline trajectory and GCLM without mean stationarity both having AR(1)CL(1) specification. Since these models allow for time-varying inter-individual differences in trajectories, we will be able to assess the effect of fitting models which do not account for this (i.e., CLPM, RI-CLPM \& Mean Stationary GCLM) on the cross-lagged path estimates. Of secondary interest, we generate data from a standard CLPM (as given in equations 2a-b) which does not account for inter-individual differences in trajectories, as well as a bivariate modified mean spline LGCM which does not exhibit an autoregressive-cross-lagged structure. This is intended to give some sense of what we might anticipate when fitting models to data which either (a) do not exhibit inter-individual differences in trajectories (as in CLPM) or (b) do not exhibit autoregressive-cross-lagged paths (as in LGCM) both in terms of model fit/selection and parameter recovery. 
Generating conditions were informed by the results from our applied examples with LSAY data. Hence, we had s=6 measurement occasions for each of our two processes with $n=3,109$ observations per generated dataset. We chose 0.3 for the smaller and 0.5 for the larger AR path conditions per process. Cross-lagged path conditions are varied in accordance to whether one process causally dominates the other (i.e., CL paths of one process are greater than CL paths of the other), or if there is no dominance (i.e., CL paths of each process are equal). We establish our non-dominance condition as CLxy=CLyx $=0.05$ and dominance condition as CLxy $=0.10>$ CLyx $=0.05$.

For GCLM, the higher unit effect variance condition is four times greater than the variance as estimated in the LSAY examples. A similar logic was used with the LGCM-SR and LGCM generating models, however, the conditions were based on the LGCM-SR variance of the slope alone, since from the LGCM perspective this is the sole component capturing time-varying inter-individual differences in trajectory. The slope variance estimates from the LGCM-SR fit to LSAY seemed a bit small ( 2 for both math and science), hence the lower variance condition was established as twice the value of the initial estimate and the higher variance condition was four times that value. The differences in the lower and higher variance conditions should be large enough to signal any notable effects that ignoring variability may have on the cross-lagged path estimates. Another condition of interest in considering cross-lagged path estimates is how closely two processes are related at the between-person level. Thus, we apply conditions for high and low correlations between slopes or unit effects. Since the correlations amongst between-person components from our LSAY fits were extremely high, these did not seem like good reference points for setting parameter values, hence we adopted standard criteria for small (0.1) and large (0.5) correlations (Cohen, 1988) to set our low and high covariance conditions in conjunction 
with respective variances from the different conditions. All other parameter values are fixed in accordance with the results from fitting the respective models to the LSAY data. With the exception that with LGCM-SR we have multiple, extremely large correlations amongst the growth curve parameters, in which case we fixed the intercept to intercept correlation as 0.5 (large), the within process intercept to slope correlation as 0.3 (medium), and the between process intercept to slope correlation as 0.1 (low). Each condition was simulated over 1,000 Monte Carlo samples.

We fit $\operatorname{AR}(1) C L(1)$ configurations of the CLPM (as given in equations 2a-b), predetermined ALT with unstructured trajectory, GCLM, Mean Stationary GCLM, LGCM-SRUGT, and RI-CLPM to each of the four generating models. Models are evaluated in terms of: conventional model fit criteria (Hu \& Bentler, 1999) for CFI, RMSEA, and SRMR; model selection with Bayes Factor (i.e., $B F_{i j} \approx e^{B I C_{i}-B I C_{j}}$ ) using criteria given in table 1; and, relative bias in cross-lagged path estimates and standard errors. In the results, for succinctness, we will focus on presenting overall bias amongst models as well as some of the more notable influences of varying conditions in the LGCM-SR and GCLM generating models on inducing bias in the cross-lagged path estimates and standard errors from fitting models which account for stable inter-individual differences yet do not account time-varying inter-individual differences ${ }^{6}$.

\footnotetext{
${ }^{6}$ All simulation codes and complete results (including convergence information, model fit/selection, and crosslagged path/standard error biases across simulation conditions for all combinations of generating and fitting models) are available upon request (send request to pws5@pitt.edu )
} 


\section{Results}

\section{Model Fit Indices and Selection}

Absolute fit indices SRMR \& RMSEA assign the lowest value to the correct model. However, conventional cut criteria would deem some non-generating models as having good fit as well. Since it accounts for model complexity, RMSEA is more conservative in determining the correct model fit than SRMR. When fitting to the GCLM data, only LGCM-SR yields, on average, an RMSEA value right on the cusp of signaling "good" fit, while SRMR signals ALT, CLPM, Mean Stationary GCLM, as well as LGCM-SR as having "good" fit. In regards to fitting to the LGCM-SR data, RMSEA does not, on average, signal any model other than LGCM-SR as having "good" fit, while SRMR suggests that GCLM, Mean Stationary GCLM, RI-CLPM, and CLPM may all exhibit "good" fit. Aside from the CLPM fit to the GCLM data, CFI would, on average, indicate all models as having good fit.

Bayes Factor does well at selecting the correct model. Exceptions occur when fitting LGCM-SR to GCLM data, and GCLM to LGCM-SR data. Specifically, when fitting to GCLM, the non-dominance condition with low unit effect covariance, high $\mathrm{x}$ unit effect variance, low $\mathrm{y}$ unit effect variance, low $\mathrm{x}$ autoregression, and high y autoregression led LGCM-SR to being erroneously chosen by Bayes Factor. When fitting to LGCM-SR, the non-dominance condition with high slope covariance and both processes being characterized by high autoregression and low slope variance, GCLM was erroneously chosen over LGCM-SR. In application, the data generating process is unknown, thus it may be informative to consider selection amongst nongenerating models. Table 3 summarizes model selection findings relating to the fitting of nongenerating models. 
Table 3. Bayes Factor based decisions for Selecting amongst Fitted Models that do not correspond to the Data Generating Model.

\begin{tabular}{|l|l|l|}
\hline \multicolumn{2}{|l|}{ Models fit to LGCM-SR } & Criteria Interpretation with Bayes Factor \\
\hline ALT & GCLM & $100 \%(\mathrm{n}=64)$ Very Strongly Favors GCLM \\
\hline ALT & RI-CLPM & $100 \%(\mathrm{n}=64)$ Very Strongly Favors ALT \\
\hline ALT & Mean Stationary & $82.81 \%(\mathrm{n}=53)$ Very Strongly Favors ALT \\
& GCLM & $15.625 \%(\mathrm{n}=10)$ Very Strongly Favors Mean Stationary GCLM \\
& & $1.6 \%(\mathrm{n}=1)$ Moderately Favors Mean Stationary GCLM \\
\hline RI-CLPM & Mean Stationary & $90.6 \%(\mathrm{n}=58)$ Very Strongly Favors Mean Stationary GCLM \\
& GCLM & $9.4 \%(\mathrm{n}=6)$ Very Strongly Favors RI-CLPM \\
\hline RI-CLPM & GCLM & $100 \%$ Very Strongly Favors GCLM \\
\hline GCLM & Mean Stationary & $100 \%$ Very Strongly Favors GCLM \\
\hline Models fit to GCLM & GCLM & \\
\hline ALT & LGCM-SR* & Criteria Interpretation with Bayes Factor \\
& & $89 \%(\mathrm{n}=57)$ Very Strongly Favors LGCM-SR \\
\hline ALT & RI-CLPM & $1.6 \%(\mathrm{n}=1)$ Very Strongly Favors ALT \\
& & $92.2 \%(\mathrm{n}=59)$ Very Strongly Favors ALT \\
& & $6.2 \%(\mathrm{n}=4)$ Very Strongly Favors RI-CLPM \\
\hline ALT & $1.6 \%(\mathrm{n}=1)$ Moderately Favors ALT \\
\hline RI-CLPM & Mean Stationary & $85.9 \%(\mathrm{n}=55)$ Very Strongly Favors ALT \\
& GCLM & $14.1 \%(\mathrm{n}=9)$ Very Strongly Favors Mean Stationary GCLM \\
\hline RI-CLPM & Mean Stationary & $76.6 \%(\mathrm{n}=49)$ Very Strongly Favors Mean Stationary GCLM \\
\hline LGCM-SR & GCLM & $21.9 \%(\mathrm{n}=14)$ Very Strongly Favors RI-CLPM \\
& & $1.6 \%(\mathrm{n}=1)$ Moderately Favors RI-CLPM \\
\hline & LGCM-SR & $90.6 \%(\mathrm{n}=58)$ Very Strongly Favors LGCM-SR \\
\hline & Mean Stationary & $90.6 \%(\mathrm{n}=58)$ Very Strongly Favors LGCM-SR \\
\hline
\end{tabular}

*9.4\% ( $\mathrm{n}=6)$ LGCM-SR couldn’t fit to GCLM

Relative Bias in cross-lagged path estimates and standard errors

Table 4 summarizes overall relative bias when fitting models ignoring time-variant interindividual differences. CLPM which ignores both time-invariant and time-variant differences tends to underestimate cross-lagged paths and overestimate standard errors. When fitting to GCLM, Mean Stationary GCLM \& RI-CLPM tend to overestimate cross-lagged paths and underestimate standard errors. When fitting to LGCM-SR, Mean Stationary GCLM tends to underestimate the cross-lagged paths and standard errors, while RI-CLPM underestimates crosslagged paths but yields essentially no bias in standard errors. 
Table 4. Overall Relative Bias in Cross-Lagged Path Estimates and Standard Errors when fitting CLPM, RI-CLPM, and Mean Stationary GCLM to data generated from LGCM-SR and GCLM.

\begin{tabular}{|l|l|l|l|}
\hline & & GCLM Generated & LGCM-SR Generated \\
\hline Fitting Model & Estimate & Mean Relative Bias (SD) & Mean Relative Bias (SD) \\
\hline CLPM & CLxy Path & $-0.29(0.41)$ & $-0.64(0.27)$ \\
& CLyx Path & $-0.34(0.47)$ & $-0.84(0.39)$ \\
& CLxy SE & $0.23(0.11)$ & $0.08(0.03)$ \\
& CLyx SE & $0.28(0.11)$ & $0.05(0.04)$ \\
\hline \multirow{5}{*}{ Mean Stationary GCLM } & CLxy Path & $0.32(0.74)$ & $-0.55(0.66)$ \\
& CLyx Path & $0.31(0.80)$ & $-1.14(0.93)$ \\
& CLxy SE & $-0.28(0.33)$ & $-0.28(0.24)$ \\
& CLyx SE & $-0.29(0.34)$ & $-1.71(0.24)$ \\
\hline RI-CLPM & CLxy Path & $0.24(1.19)$ & $-0.50(0.55)$ \\
& CLyx Path & $0.28(1.70)$ & $-0.76(0.69)$ \\
& CLxy SE & $-0.30(0.24)$ & $\sim 0(0.09)$ \\
& CLyx SE & $-0.23(0.29)$ & $\sim 0(0.086)$ \\
\hline
\end{tabular}

Table 5 shows overall relative bias when fitting to CLPM and LGCM data. When fitting to CLPM data, LGCM-SR tends to underestimate cross-lag paths while minorly overestimating the standard errors; ALT yields notable overestimation of the cross-lag paths and underestimation of the standard errors; both GCLM and Mean Stationary GCLM exhibit minor underestimation of both the cross-lag paths and standard errors; and, RI-CLPM tends to yield near zero misestimation. When fitting to LGCM $(\mathrm{CL}=0)$ : ALT and CLPM tend to yield positive crosslagged path estimates, with ALT having more "overestimation"; while RI-CLPM, GCLM and Mean Stationary GCLM lead to a mix of positive and negative mis-estimates. LGCM-SR yields essentially no misestimation of the cross-lagged paths.

Table 5. Overall Relative Bias in Cross-Lagged Path Estimates and Standard Errors when fitting GCLM, ALT, LGCM-SR, Mean Stationary GCLM, RI-CLPM, and CLPM to data generated from the CLPM (no between-person components) and LGCM (no autoregressive cross-lagged components). 


\begin{tabular}{|l|l|l|l|}
\hline & & CLPM Generated & LGCM Generated \\
\hline Fitting Model & Estimate & Mean Relative Bias $($ SD) & Mean Relative Bias (SD) \\
\hline ALT & CLxy Path & $1.487(1.19)$ & $0.45(0.04)$ \\
& CLyx Path & $1.913(1.34)$ & $0.44(0.04)$ \\
& CLxy SE & $-0.805(0.18)$ & -- \\
& CLyx SE & $-0.753(0.30)$ & -- \\
\hline GCLM & CLxy Path & $-0.007(\sim 0)$ & $0.01(0.04)$ \\
& CLyx Path & $-0.017(0.01)$ & $\sim 0(0.04)$ \\
& CLxy SE & $-0.100(0.05)$ & -- \\
& CLyx SE & $-0.092(0.05)$ & -- \\
\hline \multirow{5}{*}{ Mean Stationary GCLM } & CLxy Path & $-0.0001(\sim 0)$ & $0.01(0.08)$ \\
& CLyx Path & $-0.005(\sim 0)$ & $-0.04(0.08)$ \\
& CLxy SE & $-0.041(0.03)$ & -- \\
\hline LGCM-SR & CLyx SE & $-0.043(0.03)$ & -- \\
& CLxy Path & $-0.254(0.46)$ & $\sim 0(\sim 0)$ \\
& CLyx Path & $-0.235(0.47)$ & $\sim 0(\sim 0)$ \\
& CLxy SE & $0.025(0.08)$ & -- \\
& CLyx SE & $0.020(0.06)$ & -- \\
\hline RI-CLPM & CLxy Path & $0.001(\sim 0)$ & $0.05(0.07)$ \\
& CLyx Path & $0.002(\sim 0)$ & $\sim 0(0.09)$ \\
& CLxy SE & $0.006(0.03)$ & -- \\
& CLyx SE & $0.004(0.03)$ & -- \\
\hline CLPM & CLxy Path & $0.0005(0.004)$ & $0.03(0.02)$ \\
& CLyx Path & $-0.004(0.002)$ & $0.01(0.02)$ \\
& CLxy SE & $0.006(0.024)$ & -- \\
& CLyx SE & $0.007(0.021)$ & -- \\
\hline
\end{tabular}

As the cross-lagged path from y to $\mathrm{x}$ (CLxy) changes between dominance conditions, as one would expect, some notable patterns of bias in path estimates and standard errors emerge across dominance conditions in conjunction with slope/unit effect variance and covariance conditions when fitting models ignoring time-variant inter-individual differences. Figure 3 displays some of these notable findings. 
(a)

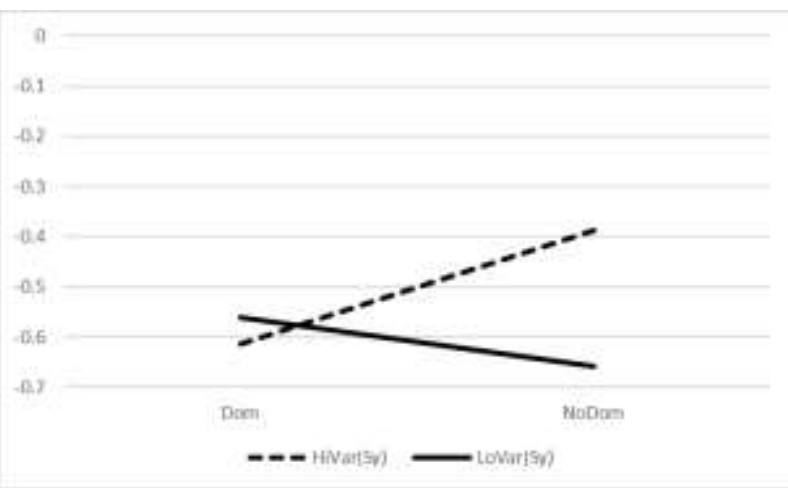

(c)

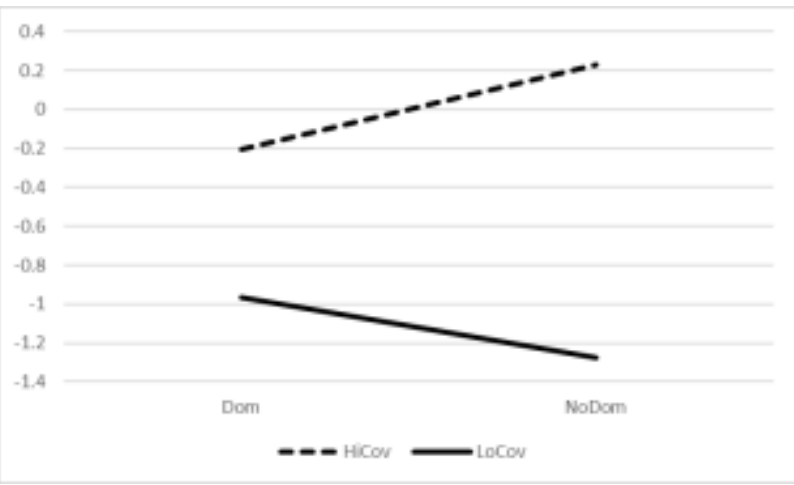

(e)

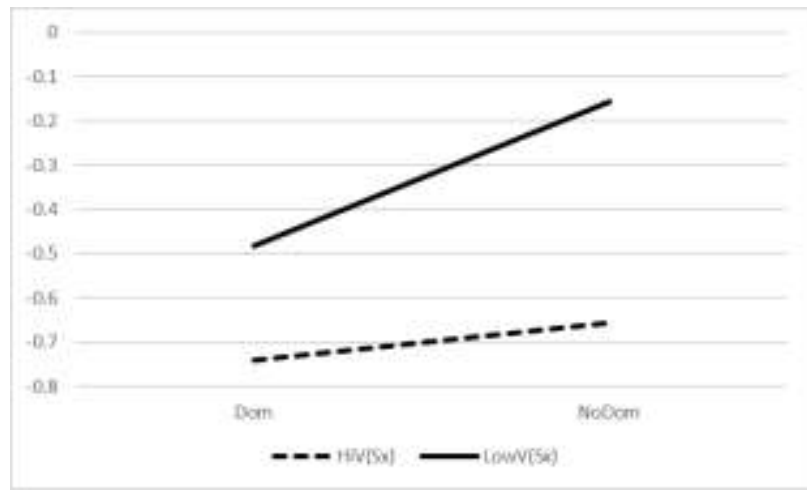

(b)

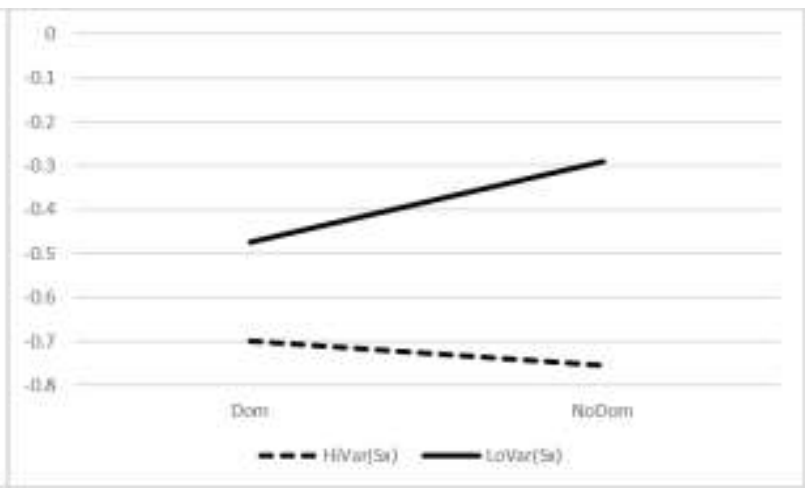

(d)

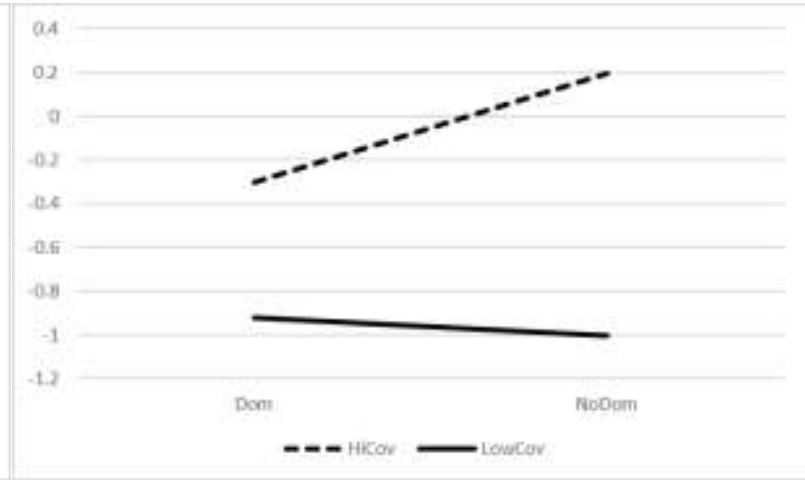

(f)

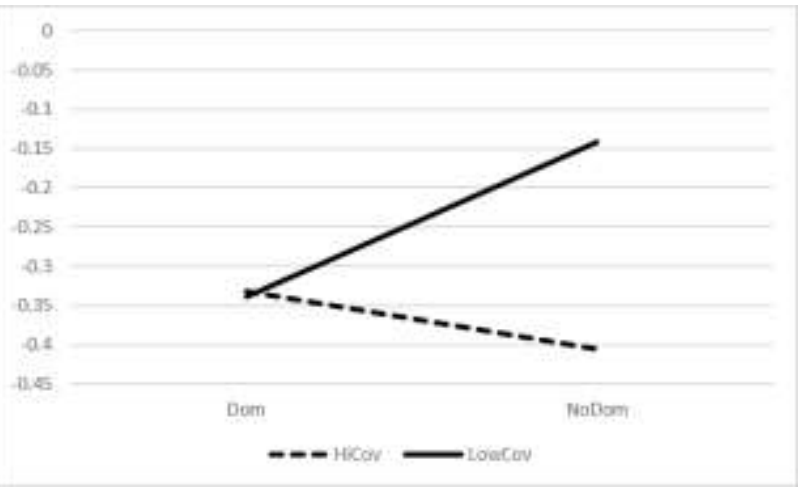

Figure 3. Effect of Dominance on Relative Bias in CLxy path estimates and standard errors across slope/unit effect variance and covariance conditions. Panel (a) Path Estimate Bias when fitting Mean Stationary GCLM to LGCM-SR across Predicting Slope Variance; Panel (b) Path Estimate Bias when fitting Mean Stationary GCLM to LGCM-SR across Outcome Slope Variance; Panel (c) Path Estimate Bias when fitting Mean Stationary GCLM to LGCM-SR across Slope Covariance; Panel (d) Path Estimate Bias when fitting RI-CLPM to LGCM-SR across Slope Covariance; Panel (e) Path Estimate Bias when fitting RI-CLPM to LGCM-SR across Outcome Slope Variance; Panel (f) Standard Error Bias when fitting RI-CLPM to GCLM across Unit Effect Covariance. 
The CLyx path estimate bias is not greatly influenced by the dominance condition when fitting the Mean Stationary GCLM to LGCM-SR data. However, the slope variances and covariance are still influential factors. Lower variance in the predicting process slope (x) leads to greater underestimation, -1.46 to -0.827 from lower to higher variance. While, variance in the outcome process (y) slope has the opposite influence, with higher variance leading to greater underestimation, -0.641 to -1.645 from lower to higher variance. Lower covariance between the slopes corresponds to greater underestimation, -1.814 to -0.473 from low to high. When fitting Mean Stationary GCLM to GCLM, relative bias in the CLyx path estimates is influenced by both autoregression and unit effect variance of the predicting process (x). Specifically, higher autoregression in the predicting process leads to greater overestimation, 0.096 to 0.524 from lower to higher autoregression. While higher unit effect of the predicting process( $\mathrm{x})$ leads to less overestimation, 0.603 to 0.017 from lower to higher.

When fitting RI-CLPM to LGCM-SR data, lower variance in the predicting slope yields greater underestimation, from -0.944 to -0.587 from lower to higher variance, while higher variance in the outcome slope corresponds to greater underestimation, from -0.47 to -1.06 for lower to higher variance. Regarding slope covariance, we find that lower covariance leads to greater underestimation of the CLyx path, from -1.33 to -0.201 for lower to higher covariance. When fitting RI-CLPM to GCLM data, interesting patterns of under- and over-estimation of the cross-lagged paths emerge across conditions (see table 6). 
Table 6. Relative Bias in Cross-lagged Path Estimates (CLxy \& CLyx) across various conditions when fitting RI-CLPM to GCLM.

\begin{tabular}{|l|l|l|l|}
\hline Path & Factor & Condition & Mean Relative Bias $($ SD) \\
\hline CLxy & ARy & Low & $0.642(1.148)$ \\
& & High & $-0.149(1.125)$ \\
\hline & ARx & Low & $0.645(1.168)$ \\
& & High & $-0.152(1.102)$ \\
\hline & Predictor(y) Unit Effect Variance & Low & $0.619(1.175)$ \\
& & High & $-0.126(1.113)$ \\
\hline & \multirow{2}{*}{ Unit Effect Covariance } & Low & $-0.303(0.743)$ \\
& & High & $0.796(1.316)$ \\
\hline & ARy & Low & $1.027(1.818)$ \\
& & High & $-0.452(1.201)$ \\
\hline & Dominance & Dominated & $0.767(1.989)$ \\
& & Not Dominated & $-0.192(1.203)$ \\
\hline & Outcome(y) Unit Effect Variance & Low & $0.721(1.441)$ \\
& & High & $-0.146(1.847)$ \\
\hline & Unit Effect Covariance & Low & $-0.488(1.231)$ \\
& & High & $1.063(1.766)$ \\
\hline
\end{tabular}

When fitting Mean Stationary GCLM to LGCM-SR data, CLxy standard errors are influenced by slope variance of the predicting process (y) with underestimation being greater in the lower variance condition, -0.367 to -0.208 from low to high, while relative bias in CLyx standard errors are influenced by slope variance in the outcome process (y), such that lower variance corresponds to more severe underestimation, -0.324 to -0.02 . Additionally, higher variance in the unit effect for the predicting process (y) leads to greater underestimation of CLxy standard errors, from 0.241 to -0.368 for lower to higher variance, and higher unit effect variance in the predicting process $(\mathrm{x})$ lead to greater underestimation of the CLyx standard errors, from -0.160 to -0.307 for lower to higher variance.

When fitting RI-CLPM to LGCM-SR data, higher slope covariance corresponds to slight underestimation of the CLxy standard errors (-0.024) while lower covariance corresponds to slight overestimation (0.026). Higher slope variance in the outcome process(x) corresponds to an overestimation of the CLxy standard error (0.074) while lower variance corresponds to an underestimation (-0.072). For the CLyx standard errors, variance in the slope for outcome(y) and 
predicting(x) process have an inverse influence on bias, from 0.018 to -0.025 and -0.05 to 0.043

for lower to higher variance for the predicting $(\mathrm{x})$ and outcome $(\mathrm{y})$ processes respectively.

\section{Discussion}

In this paper we presented on related models which explicitly aim to evaluate reciprocal relationships between two processes over time. The key distinctions highlighted pertain to whether lagged relations were modeled to the observed or residual variables and whether models accounted for inter-individual trajectory differences as time-varying or time-invariant only. These models share a representation of lagged relations but differ in terms of whether they account for inter-individual trajectory differences in a time-invariant way alone or time-varying as well. We suggest the usage of Bayes Factor as a generalized criteria for model selection, however, model fit and selection considerations were given generic addressal as secondary aims of this research, thus warrant further investigation. When considering the LSAY data examples we found that there was consensus in determining that no causal dominance was suggested by the cross-lagged paths and that most of the relationship between math and science achievement over time appeared to be at the between-person level. This latter point may be relevant when considering that LGCM-SR was selected for fitting the LSAY data relative to all of the other models, particularly in light of findings from the simulation study indicating the superiority of LGCM-SR when fitting to LGCM data, where the entire relationship between process is represented at the between person level. The magnitude of the cross-lagged path estimates was influenced by the fitted model (refer to table 1 for specifics).

From the simulation studies we found a general tendency for cross-lagged paths to be underestimated when ignoring both time-invariant and time-varying differences in individual's trajectories, while their corresponding standard errors were underestimated. However, when only 
ignoring time-varying differences an intricate pattern of under- and over-estimation emerged across models and conditions. These overall tendencies of misestimation must be interpreted in the context of how they varied across conditions. Generally, the most severe misestimation issues pertained to the cross-lagged paths. The dominance condition appeared as a significant moderator of the extent to which slope/unit effect variance and covariance induced bias in the cross-lagged path estimates. Of note, both Mean Stationary GCLM and RI-CLPM yielded severe underestimation when slope covariance of the LGCM-SR was low. While fitting the RI-CLPM to GCLM with unit effect covariance yielded severe overestimation. The various patterns of misestimation imply that it is important to consider time-varying differences in trajectory as well as the underlying conditions characterizing the relationship between two processes. For tractability, we only explored up to two-way interactions amongst conditions, while a more accurate (albeit complex) story may be revealed had we considered higher orders of interaction. Another important point worth noting is that the unit effect of the GCLM is not a perfect analog to the slope of the LGCM-SR. This is primarily due to the fact that unit effect variance contains information about both overall standing as well as change of individuals over time. It is plausible to fit more unit effects to a process that may better capture variance in change, however, this brings conceptual and empirical problems with it. Conceptually, similar to ALT, by fitting the CLPM to the observed variables the unit effects do not truly function as growth curve parameters. Empirically, attempts to establish such a model resulted in either convergence problems or estimates that were hard to make sense of. This line of inquiry could prove valuable for future research, because of the shared features between GCLM and ALT, this line of inquiry would likely be well-informed by the literature building our understanding of ALT. ${ }^{7}$

\footnotetext{
${ }^{7}$ All MPlus and R codes used in this study are available upon request from pws5@pitt.edu.
} 


\section{References}

Allison, P. D., Williams, R., \& Moral-Benito, E. (2017). Maximum likelihood for cross-lagged panel models with fixed effects. Socius, 3, 2378023117710578.

Bollen, K. A., \& Zimmer, C. (2010). An overview of the autoregressive latent trajectory (ALT) model. In Longitudinal research with latent variables (pp. 153-176). Springer, Berlin, Heidelberg.

Bollen, K. A., \& Curran, P. J. (2004). Autoregressive latent trajectory (ALT) models a synthesis of two traditions. Sociological Methods \& Research, 32(3), 336-383.

Chi, E. M., \& Reinsel, G. C. (1989). Models for longitudinal data with random effects and AR (1) errors. Journal of the American Statistical Association, 84(406), 452-459.

Clark, D. A., Nuttall, A. K., \& Bowles, R. (2020, March 4). Process Distinguishability in Hybrid Longitudinal Models: Number of Time Points, Misspecification, Bias, and Change. https://doi.org/10.31234/osf.io/5beqm

Clark, D. A., Nuttall, A. K., \& Bowles, R. P. (2018). Misspecification in latent change score models: Consequences for parameter estimation, model evaluation, and predicting change. Multivariate behavioral research, 53(2), 172-189.

Cohen, Jacob. (1988). Statistical Power analysis for the Behavioral Sciences, 2nd ed. Lawrence Erlbaum Associates

Curran, P. J., \& Bollen, K. A. (2001). The best of both worlds: Combining autoregressive and latent curve models. In L. M. Collins \& A. G. Sayer (Eds.), Decade of behavior. New methods for the analysis of change (p. 107-135). American Psychological Association. 
Curran, P. J., Howard, A. L., Bainter, S. A., Lane, S. T., \& McGinley, J. S. (2014). The separation of between-person and within-person components of individual change over time: A latent curve model with structured residuals. Journal of Consulting and Clinical Psychology, 82(5), 879-894.

Eichler, M. (2013). Causal inference with multiple time series: principles and problems. Philosophical Transactions of the Royal Society A, 371, 2011.0613.

Granger, C. W. (1969). Investigating causal relations by econometric models and cross-spectral methods. Econometrica: Journal of the Econometric Society, 424-438.

Granger, C. W. (1980). Testing for causality: a personal viewpoint. Journal of Economic Dynamics and control, 2, 329-352.

Hallquist, M. N. \& Wiley, J. F. (2018). MplusAutomation: An R Package for Facilitating LargeScale Latent Variable Analyses in Mplus. Structural Equation Modeling: a multidisciplinary journal, 1-18. doi:10.1080/10705511.2017.1402334.

Hamaker, E. L. (2005). Conditions for the equivalence of the autoregressive latent trajectory model and a latent growth curve model with autoregressive disturbances. Sociological Methods \& Research, 33(3), 404-416.

Hamaker, E. L., Kuiper, R. M., \& Grasman, R. P. (2015). A critique of the cross-lagged panel model. Psychological methods, 20(1), 102-116.

Hancock, G. R., \& Lawrence, F. R. (2006). Using latent growth models to evaluate longitudinal change. Structural equation modeling: A second course, 171-196. 
Hu, L. T., \& Bentler, P. M. (1999). Cutoff criteria for fit indexes in covariance structure analysis: Conventional criteria versus new alternatives. Structural equation modeling: $a$ multidisciplinary journal, 6(1), 1-55.

Jeffreys, H. (1998). The theory of probability. UK: Oxford University Press.

Jongerling, J., \& Hamaker, E. L. (2011). On the trajectories of the predetermined ALT model: What are we really modeling?. Structural Equation Modeling: A Multidisciplinary Journal, 18(3), 370-382.

Jöreskog, K.G. (1970). A general method for analysis of covariance structures. Biometrika, $57,239-251$.

Jöreskog, K.G. \& Sörbom, D. (1979). Advances in factor analysis and structural equation models. Cambridge, MA: Abt Books.

Kass, R. E., \& Raftery, A. E. (1995). Bayes factors. Journal of the American Statistical Association, 90(430), 773-795.

Lee, S., Sriutaisuk, S., \& Kim, H. (2020). Using the Tidyverse Package in R for Simulation Studies in SEM. Structural Equation Modeling: A Multidisciplinary Journal, 27(3), 468482.

McArdle, J. J., \& Hamagami, F. (1991). Modeling incomplete longitudinal and cross-sectional data using latent growth structural models. In L. M. Collins \& J. C. Horn (Eds.), Best methods for tile analysis of change (pp. 276-304). Washington, DC: American Psychological Association.

Meredith, W., \& Tisak, J. (1990). Latent curve analysis. Psychometrika, 55(1), 107-122 
Miller, Jon D. Longitudinal Study of American Youth (LSAY), 1987-1994. Ann Arbor, MI: Inter-university Consortium for Political and Social Research [distributor], 2016-03-24.

Muthén, L. K., \& Muthén, B. O. (1998-2017). MPlus User's Guide. Eighth Edition. Los Angeles, CA: Muthén \& Muthén

Ou, L., Chow, S. M., Ji, L., \& Molenaar, P. C. (2017). (Re) evaluating the implications of the autoregressive latent trajectory model through likelihood ratio tests of its initial conditions. Multivariate behavioral research, 52(2), 178-199.

Preacher, K. J., Wichman, A. L., MacCallum, R. C., \& Briggs, N. E. (2008). Latent growth curve modeling (Quantitative Applications in the Social Sciences, No. 157). SAGE.

Raftery, A. E. (1999). Bayes factors and BIC: Comment on "A critique of the Bayesian information criterion for model selection". Sociological Methods \& Research, 27(3), 411-427.

R Core Team (2019). R: A language and environment for statistical computing. R Foundation for Statistical Computing, Vienna, Austria. URL https://www.R-project.org/.

Usami, S., Murayama, K., \& Hamaker, E. L. (2019). A unified framework of longitudinal models to examine reciprocal relations. Psychological methods.

Venables, W. N. \& Ripley, B. D. (2002) Modern Applied Statistics with S, Fourth Edition. Springer: New York.

Voelkle, M. C. (2008). Reconsidering the use of autoregressive latent trajectory (ALT) models. Multivariate Behavioral Research, 43(4), 564-591.

Wickham, H. (2017). tidyverse: Easily Install and Load the 'Tidyverse'. R package version 1.2.1. https://CRAN.R-project.org/package=tidyverse 
Zyphur, M. J., Allison, P. D., Tay, L., Voelkle, M. C., Preacher, K. J., Zhang, Z., Hamaker, E.L., Shamsollahi, A., Pierides, D.C., Koval, P. \& Diener, E. (2019a). From Data to Causes I: Building A General Cross-laggedged Panel Model (GCLM). Organizational Research Methods. https://doi.org/10.1177/1094428119847278

Zyphur, M. J., Voelkle, M. C., Tay, L., Allison, P. D., Preacher, K. J., Zhang, Z., Hamaker, E.L., Shamsollahi, A., Pierides, D.C., Koval, P., \& Diener, E. (2019b). From Data to Causes II: Comparing Approaches to Panel Data Analysis. Organizational Research Methods. https://doi.org/10.1177/1094428119847280 